ISSN 1997-5902

\title{
Evaluation du potentiel antimicrobien et de la toxicité des extraits de Jatropha multifida Linn, (Euphorbiaceae)
}

\author{
Amégninou Agban1, Yao Hoekou1, Passimna Pissang1, Tchadjobo Tchacondo1, Komlan Batawila² \\ ${ }^{1}$ Laboratoire des Sciences Biomédicales, Alimentaires et de Santé Environnementale (LaSBASE), Ecole Supérieure \\ des Techniques Biologiques et Alimentaires (ESTBA), Université de Lomé, Togo. \\ 2Laboratoire de Botanique et Ecologie Végétale, Faculté des Sciences (FDS), Université de Lomé, Togo. \\ Corresponding Author: Dr Yao HOEKOU, Département des Analyses Médicales et Biologiques, Ecole Supérieure \\ des Techniques Biologiques et Alimentaires (ESTBA), Université de Lomé, 01 BP 1515 Lomé, Togo. E-mail: \\ yhoekou@gmail.com
}

Original submitted in on $11^{\text {th }}$ May 2020. Published online at www.m.elewa.org/journals/ on $31^{\text {st }}$ July 2020 https://doi.org/10.35759/JABs.151.4

\section{RÉSUMÉ}

Objectif : L'objectif de ce travail était d'évaluer in vitro l'activité antimicrobienne des extraits de feuilles et tige de Jatropha multifida sur la croissance de Candida albicans, Escherichia coli et Staphylococcus aureus, puis d'évaluer in vivo la toxicité de cette plante.

Méthodologie et résultats : Les méthodes de diffusion en milieu gélosé et de microdilution en milieu liquide ont été utilisées pour évaluer l'effet antimicrobien. Une étude en subaigüe était réalisée afin d'explorer les effets toxiques de l'extrait aqueux des feuilles. Les résultats des tests antimicrobiens montrent une activité des extraits de feuilles et tige de $J$. multifida sur la croissance des souches utilisées avec des diamètres de zones d'inhibition allant de 8 à $25 \mathrm{~mm}$ et des concentrations minimales inhibitrices (CMI) variant de 0,039 $\mathrm{mg} / \mathrm{mL}$ à $1,25 \mathrm{mg} / \mathrm{mL}$ à l'exception des souches de $E$. coli qui sont résistantes aux extraits de la tige. L'administration en subaigüe de l'extrait aqueux des feuilles de J. multifida à la dose de $600 \mathrm{mg} / \mathrm{kg}$ entraîne une perte significative de poids chez les souris.

Conclusion et applications des résultats : Les extraits aqueux, éthanolique et hydroéthanolique des feuilles et tige de J. multifida possèdent d'activité antimicrobienne et pourraient être utilisés dans le traitement des Candidoses à $C$. albicans et des infections à $S$. aureus. Mais l'essai de toxicité subaigüe montre que l'extrait aqueux de la plante serait toxique. Des études toxicologiques approfondies restent donc nécessaires sur ces extraits afin de mieux élucider leur inocuité.

Mots-clés : Jatropha multifida, extraits de feuilles et de tige, activités antifongique et antibactérienne, toxicité. 


\section{Evaluation of antimicrobial potential and toxicity of Jatropha multifida Linn, (Euphorbiaceae)} extracts

\section{ABSTRACT}

Objective: The objective of this study was to evaluate in vitro the antimicrobial activity of leaves and stem of Jatropha multifida extracts against Candida albicans, Escherichia coli and Staphylococcus aureus, and then to evaluate in vivo the toxicity of this plant.

Methodology and Results: The agar well-diffusion and the NCCLS broth microdilution methods were used to assess the antimicrobial effect. A subacute study was carried out to explore the toxic effects of the aqueous extract of the leaves. The results of the antimicrobial tests show an activity of the extracts of leaves and stems of $\mathrm{J}$. multifida on the growth of the strains used with diameters of inhibitory zones ranging from 8 to $25 \mathrm{~mm}$ and minimum inhibitory concentrations (MIC) varying from $0.039 \mathrm{mg} / \mathrm{mL}$ to $1.25 \mathrm{mg} / \mathrm{mL}$ exception $E$. coli strains which are resistant to extracts from the stem. Subacute administration of the aqueous extract of the leaves of $\mathrm{J}$. multifida at a dose of $600 \mathrm{mg} / \mathrm{kg}$ leads to a significant loss of weight in the mice.

Conclusion and application of findings: The aqueous, ethanolic and hydroethanolic extracts of the leaves and stem of $\mathrm{J}$. multifida have antimicrobial activity and could be used in the treatment of Candidiasis and bacterial infections due respectively to $C$. albicans and $S$. aureus. But the subacute toxicity test shows that the aqueous extract of the plant would be toxic. Extensive toxicological studies therefore remain necessary on these extracts in order to better elucidate their safety.

Keywords: Jatropha multifida extracts of leaves and stem, antifungal and antibacterial activities, toxicity.

\section{INTRODUCTION}

Pour faire face aux besoins de soins de santé, près de $80 \%$ de la population togolaise à l'instar de l'ensemble des pays au Sud du Sahara font recours à la médecine traditionnelle (OMS, 2013). Ceci n'est pas seulement dû à leurs cultures mais aussi à l'inégale répartition des centres de santé sur les territoires nationaux, aux difficultés liées aux déplacements, au coût élevé des prestations et médicaments conventionnels, et aux facteurs socio-économiques (Akharaiyi \& Boboye, 2010, Agban et al., 2012). Cette médecine traditionnelle se rapporte aux pratiques, méthodes, savoir en matière de santé qui impliquent, entre autres, l'usage à des fins médicales de plantes pour soigner, diagnostiquer et prévenir les maladies ou préserver la santé (Kedziers \& Jouvelet, 2006). Parmi les problèmes de santé, un intérêt particulier est porté aux maladies infectieuses car ces maladies sont à l'origine du tiers de la mortalité mondiale et sont plus fréquentes dans les pays pauvres où une personne sur deux en meurt prématurément (Tittikpina et al., 2013). Des investigations ethnobotaniques ont montré que plusieurs plantes sont utilisées en médecine traditionnelle pour le traitement des maladies infectieuses (Hoekou et al., 2016). Une des plantes utilisées pour traiter des infections fongiques, réquisitionnée lors des enquêtes, est Jatropha multifida. C'est un arbuste succulent, de la famille des Euphorbiaceae, originaire d'Amérique tropicale (du Mexique au Brésil). II est connu sous le nom de noisette purgative dans les Antilles française (Jacques, 2002). Au Mexique, les jeunes feuilles sont consommées comme légume et les graines purgatives sont utilisées en médecine traditionnelle. En Tanzanie, les guérisseurs l'emploient contre les infections fongiques. En médecine chinoise l'écorce et les feuilles sont utilisées contre les démangeaisons (Hamza et al., 2006). Malgré ces usages en médecine traditionnelle, peu de travaux ont confirmé scientifiquement les activités biologiques de cette plante (Dougnon et al., 2012 ; Falodun et al., 2014). C'est dans cette optique que la présente étude a été entreprise en vue d'évaluer les activités antifongiques et antibactériennes des extraits de la plante et d'étudier ensuite sa toxicité. 


\section{MATÉRIEL ET MÉTHODES}

Matériel végétal : Le matériel végétal est constitué des feuilles et tiges de Jatropha multifida. Ces organes sont récoltés en mars 2014 à Vogan dans la région maritime du Togo après enquête ethnobotanique auprès des tradithérapeutes de la localité. La plante a été identifiée et confirmée au laboratoire de Botanique et Ecologie Végétale de la Faculté des Sciences de l'Université de Lomé.

Souches microbiennes utilisées: Les microorganismes utilisés pour les tests sont des souches cliniques isolées aux laboratoires de bactériologie du Centre Hospitalier Universitaire du Campus de Lomé et de l'Institut National d'Hygiène de Lomé (Togo). II s'agit de Staphylococcus aureus, Escherichia coli et Candida albicans. Les souches de C. albicans ont été isolées des prélèvements génitaux et celles de $S$. aureus et $E$. coli des urines.

Traitement du matériel végétal et extraction: Les organes de plante récoltés ont été lavés puis découpés en petits morceaux et séchés au laboratoire à la température ambiante à l'abri de la lumière vive pendant 15 jours. Ils ont été ensuite pulvérisés à la moulinette de marque RETSCH, type SK 100. Les poudres obtenues ont servi à l'extraction. L'extraction a été réalisée par épuisement de solvant à polarité croissante dont le choix a été basé sur les solvants utilisés par les tradithérapeutes lors de leur préparation. Cinquante (50) g de poudre de chaque organe sont mis dans un mélange de $250 \mathrm{~mL}$ d'éther et $250 \mathrm{~mL}$ d'acétone. Le mélange a été agité pendant 10 à $15 \mathrm{mn}$ afin d'extraire et d'éliminer d'abord la chlorophylle et ses dérivés. Le mélange est filtré et le filtrat éliminé, puis l'extraction a été poursuivie sur le résidu. Le système de solvants : acétate d'éthyle- chloroforme, chloroforme, chloroforme-éthanol, éthanol, éthanol-eau et eau, a été utilisé suivant les travaux antérieurs de Kaouadji et al. (1986) et Agban et al. (2012). Chaque extrait obtenu est évaporé au rotavapor pour obtenir des extraits secs.

Criblage phytochimique : Le criblage phytochimique a consisté à mettre en évidence dans les extraits obtenus, les alcaloïdes, les flavonoïdes, les tanins et les saponines. II est fait par une analyse phytochimique qualitative sommaire à partir des tests de coloration suivant Harbone (1998).

Tests antimicrobiens : Les tests de sensibilité ont été réalisés sur gélose Mueller Hinton et Sabouraud Chloramphénicol suivant les recommandations du Comité de l'Antibiogramme de la Société Française de Microbiologie (CASFM, 2014). Les microorganismes issus d'une culture de 18 à 24 heures incubés à $37^{\circ} \mathrm{C}$ ont été suspendus dans de l'eau physiologique à une turbidité correspondante au Mac Farland 0.5 (approximativement $10^{8}$ cellules $/ \mathrm{mL}$ ). Cette suspension a été utilisée pour ensemencer des boîtes de Pétri par écouvillonnage. Les extraits éthanoliques et hydroéthanoliques ont été dissouts dans du diméthylsulfoxide (DMSO) et dilués avec de l'eau distillée stérile à une concentration finale de $1 \%$ de DMSO. Les extraits aqueux ont été dissouts dans de l'eau distillée stérile. Des puits de $6 \mathrm{~mm}$ de diamètre ont été faits dans la gélose et ces puits ont été ensuite inoculés avec $50 \mu \mathrm{L}$ d'extraits à la concentration de 20 $\mathrm{mg} / \mathrm{mL}$. La gentamicine $(15 \mu \mathrm{g} / \mathrm{mL})$ pour les bactéries, la nystatine $(100 \mu \mathrm{g} / \mathrm{mL})$ pour $C$. albicans et le DMSO à $1 \%$ dans de l'eau distillée stérile ont été utilisés respectivement comme contrôles positif et négatif. Les boîtes ont été ensuite laissées à la température ambiante pendant 1 heure pour la prédiffusion puis incubées à $37^{\circ} \mathrm{C}$ pendant 18 à 24 heures. L'activité antimicrobienne a été estimée par la mesure à la règle graduée, du diamètre de la zone d'inhibition autour des puits. Les Concentrations Minimales Inhibitrices (CMI) ont été déterminées en utilisant la technique de microdilution avec du bouillon Mueller Hinton pour les bactéries, et du bouillon Sabouraud pour C. albicans (NCCLS, 2003). Les suspensions microbiennes ont été diluées au 1/100 avec du bouillon et distribuées dans les microplaques de 96 puits contenant une gamme de concentrations de chaque extrait allant de 10 à 0,0195 $\mathrm{mg} / \mathrm{mL}$ pour chaque extrait. Les inocula déterminés par le comptage des colonies à partir des puits témoins sans extraits ont été approximativement de $10^{5}$ $\mathrm{UFC} / \mathrm{mL}$. Les plaques ont été incubées à $37^{\circ} \mathrm{C}$ pendant 24 heures pour les bactéries et 48 heures pour la levure. La CMI, déterminée à l'œil nu, a été définie comme la concentration minimale d'extrait pour laquelle on n'observe pas de croissance visible à l'œil nu.

Etude toxicologique : Le décocté aqueux a été utilisé pour l'essai toxicologique car il a été la forme galénique la plus utilisée par les tradipraticiens.

Animaux et synchronisation : Les souris mâles Swiss albinos âgées de 6 à 8 semaines ont été utilisées. Elles ont été réparties en deux lots de 10 et placées dans des cages en plastique. Les animaux ont été synchronisés pendant 2 semaines avant le début du traitement, par un cycle de $12 \mathrm{~h}$ de lumière et $12 \mathrm{~h}$ d'obscurité. L'eau et les aliments sont laissés ad libitum. La température de la salle est maintenue constante $\left(23 \pm 2^{\circ} \mathrm{C}\right)$ ainsi que l'humidité $(65 \pm 10 \%)$. 
Traitement des souris : Les souris ont été mises à jeun pendant 4 heures avant le début du traitement. Le premier groupe (Témoin) a été traité à l'eau distillée et le second a reçu une dose quotidienne de $600 \mathrm{mg} / \mathrm{kg}$ d'extrait aqueux de feuilles de J. multifida pendant 10 jours. L'eau distillée ainsi que l'extrait aqueux ont été administrés aux souris par voie orale. Une heure après chaque traitement, les souris ont été nourries et abreuvées. Le poids corporel des souris et la consommation alimentaire ont été utilisés comme

\section{RÉSULTATS}

Rendements d'extractions : Le tableau 1 présente les rendements d'extractions des feuilles et tige de $J$. multifida. Les meilleurs rendements ont été obtenus indices de toxicité pour évaluer la toxicité de l'extrait (Pissang et al., 2018).

Analyse des résultats : Les résultats ont été traités à l'aide d'Excel 2013. Les tests ANOVA un facteur suivi de la comparaison multiple de Tukey ont été effectués à l'aide du logiciel SPSS 22.0 (Chicago, USA) afin de prouver l'existence ou non d'une différence significative entre le groupe ayant reçu l'extrait et le groupe contrôle $(p<0,05$ sont considérées significatives).

avec les extraits hydroéthanoliques $(20,5 \%$ pour la tige et $16,6 \%$ pour les feuilles).

Tableau 1 : Rendements des extraits de feuilles et de tige de J. multifida

\begin{tabular}{l|c|c|c}
\hline \multirow{2}{*}{ Organes } & \multicolumn{3}{|c}{ Rendements (\%) } \\
\cline { 2 - 4 } & Extraits aqueux & Extraits hydroéthanoliques & Extraits éthanoliques \\
\hline Feuilles & 13,5 & 16,6 & 11,7 \\
Tige & 13,2 & 20,5 & 14,2 \\
\hline
\end{tabular}

Composés phytochimiques des extraits : L'examen phytochimique a révélé la présence de tanins et des flavonoïdes dans tous les extraits de feuilles et tiges de J. multifida. Les alcaloïdes ont été présents dans les extraits de feuilles alors qu'ils sont absents dans les extraits de tige. Les saponines ont été absentes dans tous les extraits testés (tableau 2).

Tableau 2 : Résultat des tests phytochimiques

\begin{tabular}{l|c|c|c|c|c|c}
\hline \multirow{2}{*}{ Composés } & \multicolumn{3}{|c|}{ Feuilles } & \multicolumn{3}{c}{ Tige } \\
\cline { 2 - 7 } & Aq & HE & Eth & Aq & HE & Eth \\
\hline Tanins & + & + & + & + & + & + \\
Flavonoïdes & + & + & + & + & + & + \\
Alcaloïdes & + & + & + & - & - & - \\
Saponines & - & - & - & - & - & -
\end{tabular}

$+=$ présence $;-=$ absence $; \mathrm{Aq}=$ extrait aqueux $; \mathrm{HE}=$ extrait hydroéthanolique $;$ Eth $=$ extrait éthanolique.

Tests antimicrobiens: Les résultats des tests antimicrobiens des extraits testés ont été consignés aux tableaux 3 et 4 . Le tableau 3 présente les diamètres des zones d'inhibition des différents extraits. Ces diamètres ont varié de 8 à $25 \mathrm{~mm}$ pour les extraits de feuilles et de 0 à $17 \mathrm{~mm}$ pour les extraits de tige. $E$. coli a été moins sensible aux extraits de feuilles avec des diamètres de zones d'inhibition allant de 8 à $10 \mathrm{~mm}$ et résistant aux extraits de tige de la plante. $C$. albicans a été le germe le plus sensible avec des diamètres variant de 22 à $25 \mathrm{~mm}$ pour les extraits de feuilles et de 13 à $17 \mathrm{~mm}$ pour les extraits de tige. 
Tableau 3 : Diamètres des zones d'inhibition des extraits testés

\begin{tabular}{|c|c|c|c|c|}
\hline \multirow[b]{2}{*}{ Organes } & \multirow[b]{2}{*}{ Extraits } & \multicolumn{3}{|c|}{ Diamètres des zones d'inhibition (en mm) } \\
\hline & & S. aureus & E. coli & C. albicans \\
\hline \multirow[b]{3}{*}{ Feuilles } & $\mathrm{Aq}$ & 12 & 8 & 24 \\
\hline & $\mathrm{HE}$ & 15 & 8 & 22 \\
\hline & Eth & 19 & 10 & 25 \\
\hline & $\mathrm{Aq}$ & 14 & 0 & 15 \\
\hline & $\mathrm{HE}$ & 12 & 0 & 17 \\
\hline Tige & Eth & 12 & 0 & 13 \\
\hline \multirow{3}{*}{\multicolumn{2}{|c|}{$\begin{array}{c}\text { Gentamicine }(15 \mu \mathrm{g} / \mathrm{mL}) \\
\text { Nystatine }(100 \mu \mathrm{g} / \mathrm{mL}) \\
\text { DMSO } 1 \%\end{array}$}} & 28 & 22 & NT \\
\hline & & NT & NT & 32 \\
\hline & & 0 & 0 & 0 \\
\hline
\end{tabular}

$\mathrm{Aq}=$ extrait aqueux $; \mathrm{HE}=$ extrait hydroéthanolique $; \mathrm{Eth}=$ extrait éthanolique, NT= non testé

Le tableau 4 indique les concentrations minimales inhibitrices (CMI) des extraits testés. Les CMI ont varié de $0,039 \mathrm{mg} / \mathrm{mL}$ à $1,25 \mathrm{mg} / \mathrm{mL}$. Les meilleures CMl ont été obtenues avec les extraits de feuilles sur la croissance de $C$. albicans.

Tableau 4 : Concentrations minimales inhibitrices (CMI) des extraits de J. multifida testés

\begin{tabular}{l|c|c|c|c}
\hline \multirow{2}{*}{ Organes } & \multirow{3}{*}{ Extraits } & S. aureus & E. coli & C. albicans \\
\cline { 3 - 5 } Feuilles & $\mathrm{Aq}$ & 0,625 & $>10$ & 0,039 \\
& $\mathrm{HE}$ & 0,625 & $>10$ & 0,039 \\
& $\mathrm{Eth}$ & 0,312 & $>10$ & 0,039 \\
\hline \multirow{3}{*}{ Tige } & $\mathrm{Aq}$ & 1,25 & $\mathrm{nd}$ & 0,312 \\
& $\mathrm{HE}$ & 1,25 & $\mathrm{nd}$ & 0,312 \\
& $\mathrm{Eth}$ & 1,25 & $\mathrm{nd}$ & 0,312 \\
\hline
\end{tabular}

nd : non déterminée

Etude toxicologique de l'extrait aqueux des feuilles de J. multifida

Effet du traitement sur la consommation alimentaire des souris : La figure 1 montre l'effet des extraits sur la consommation journalière des souris. Dans les 48 heures suivant le début du traitement, on observe que les souris traitées n'ont pas pu consommer. On remarque après 48 heures que le traitement subaigu continu par l'extrait aqueux de feuilles de J. multifida a entrainé une augmentation de la consommation chez les souris traitées comparativement aux souris du lot témoin. La baisse de la consommation alimentaire observée dans les 48 heures serait due au stress. Cette augmentation se stabilise même si le traitement continue. 


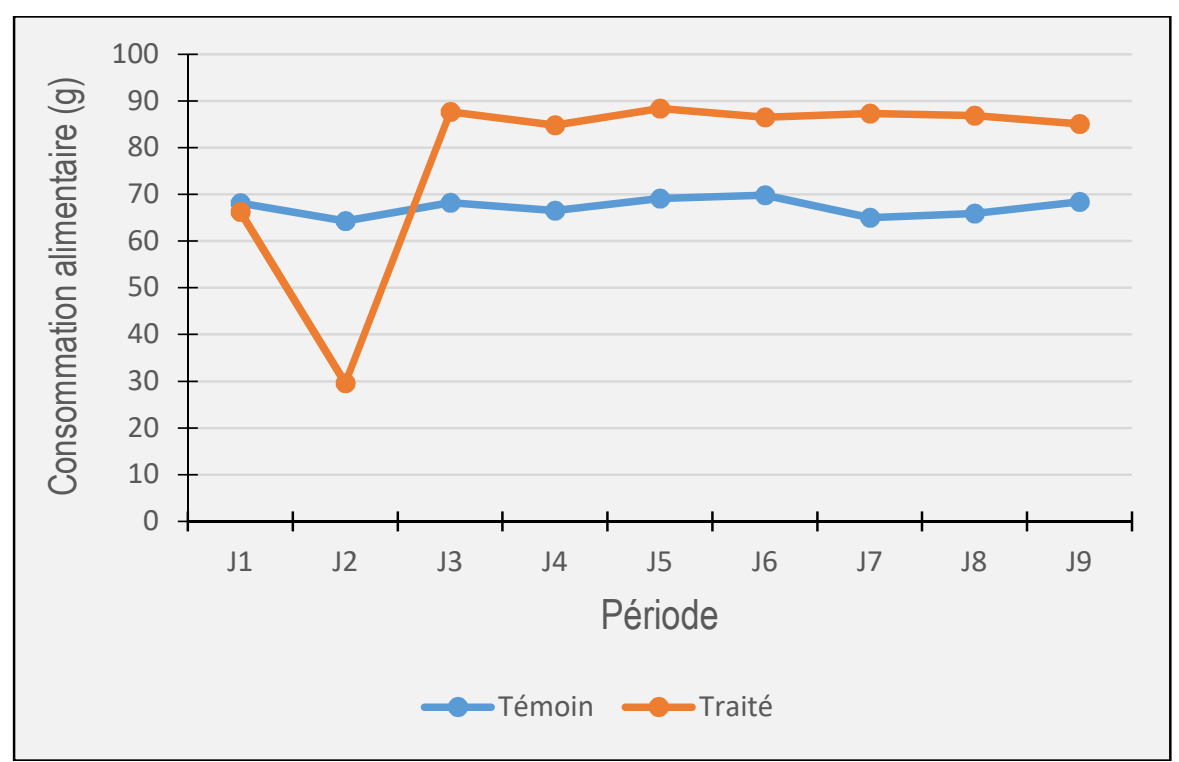

Figure 1 : Evolution de la consommation alimentaire des souris pendant le traitement

Effet du traitement sur le poids corporel des souris : La figure 2 indique l'effet du traitement sur le poids corporel des souris après 10 jours de traitement. Les souris du lot témoin ont gagné en moyenne $3,77 \mathrm{~g}$ de poids alors que celles traitées en ont perdu $5,15 \mathrm{~g}$. L'extrait a entrainé donc une perte significative de poids (seuil de $\alpha=0,05$ ) chez les souris traitées.

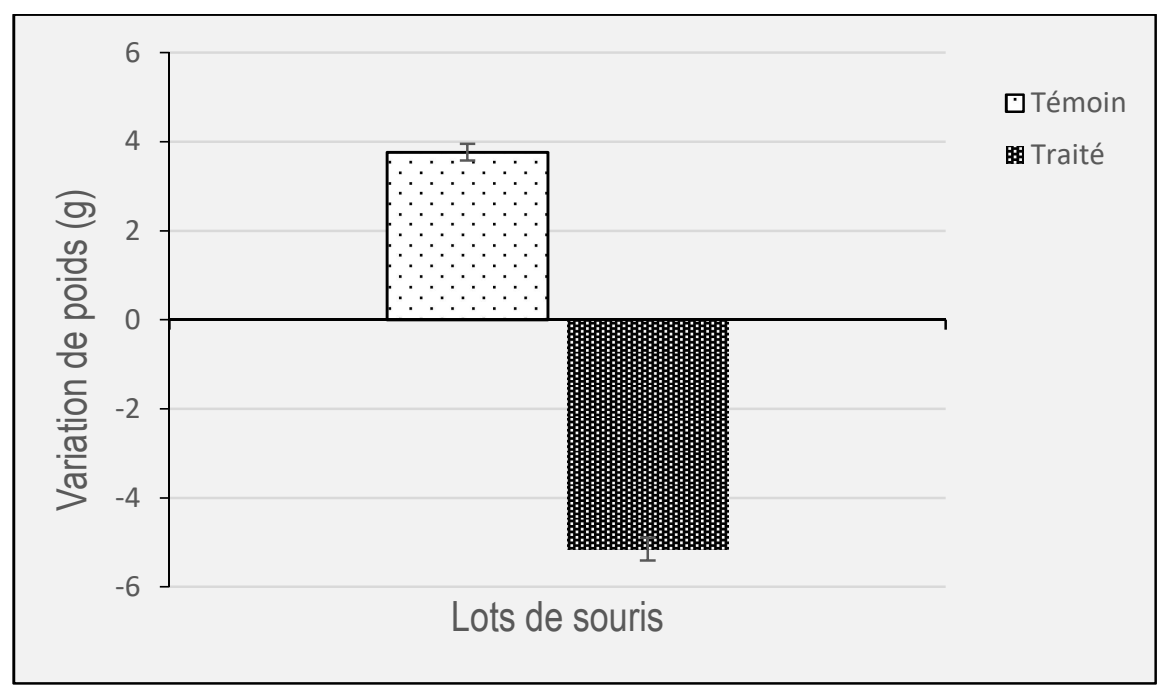

Figure 2 : Variation du poids des souris après 10 jours de traitement

\section{DISCUSSION}

L'objectif principal était d'évaluer les effets antimicrobiens et toxiques des extraits de feuilles et tige de Jatropha multifida. Le choix des feuilles surtout de cette plante comme matériel d'étude était lié à leurs usages traditionnels courant pour traiter les candidoses par les tradipraticiens de la région maritime du Togo. Les tests antimicrobiens étaient conduits sur $C$. albicans, $S$. aureus et $E$. coli, tous fréquemment isolés en pathologie humaine. Des enquêtes ethnobotaniques ont indiqué que les tradithérapeutes utilisent très souvent dans leur préparation l'eau et l'alcool (Adesola \& Adetunji, 2007). Ces indications ont orienté l'extraction qui a été réalisée en utilisant comme solvants l'éthanol, le mélange éthanol/eau et l'eau après avoir éliminé les chlorophylles et leurs dérivés. Trois sortes d'extraits ont été obtenus par épuisement de solvants à polarité croissante : extraits éthanoïques de feuille et de tige, extraits hydroethanoliques de 
feuilles et de tige, extraits aqueux de feuille et de tige. L'éthanol et surtout l'eau sont des solvants polaires et les produits extraits par eux sont des composés polaires. En effet l'analyse phytochimique des extraits révèle la présence de flavonoïdes, de tanins qui sont des composés phénoliques solubles dans l'eau, et des alcaloïdes en faible quantité, moins polaires et peu solubles dans l'eau (Bruneton, 2009). Les tests antimicrobiens ont montré que les extraits testés inhibent la croissance de toutes les souches utilisées à l'exception des extraits de tige qui sont inactifs sur les souches de $E$. coli. Les souches de $C$. albicans sont plus sensibles aux extraits testés. Les extraits de feuilles, plus actifs que ceux de la tige, ont une activité faible ou presque nulle sur les souches de $E$. coli. De ces observations, il ressort que les extraits testés sont actifs sur les microorganismes à Gram positif et inactifs sur ceux à Gram négatif. Des études antérieures ont montré que les bactéries à Gram positif sont généralement les plus sensibles aux extraits végétaux (Fennell et al., 2004). Ceci pourrait s'expliquer par la différence de la composition de la paroi des bactéries. En effet, les bactéries Gram positives ont une paroi riche en peptidoglycane favorisant la pénétration des phytomolécules qui pourraient atteindre facilement leurs cibles intracellulaires. Par contre, les bactéries Gram négatives avec leur membrane externe composée des phospholipides qui interfèrerait avec les phytomolécules, rend leur passage à travers la paroi difficile surtout les composés hydrophobes (Soundararajan et al., 2012). Les extraits de feuilles testés renferment de tanins, d'alcaloïdes et de flavonoïdes alors que ceux de tige ne renferment que de flavonoïdes et de tanins. Ceci pourrait expliquer la différence d'activité observée entre ces deux organes de J. multifida. Falodun et al. (2014) ont isolé des composés terpénoïdes de la tige de J. multifida qui ont des activités antifongiques sur des souches de références de C. albicans (ATCC 90028), C. glabrata (ATCC 90030), C. krusei (ATCC 6258), Cryptococcus neoformans (ATCC 90113) et Aspergillus fumigatus

\section{CONCLUSION ET APPLICATION DES RÉSULTATS}

Les extraits aqueux, éthanolique et hydroéthanolique de feuilles et tige de J. multifida sont actifs sur $C$. albicans et $S$. aureus. Ce résultat démontre le potentiel antimicrobien des feuilles et tige de $\mathrm{J}$. multifida et contribue à la justification de l'usage de la plante contre les candidoses et les infections staphylococciques. Mais les résultats des tests de toxicité in vivo sur des
(ATCC 204305), et antibactériennes sur S. aureus (ATCC 29213), S. aureus meti-R (ATCC 33591), Pseudomonas aeruginosa (ATCC 27853) et Mycobacterium intracellulare (ATCC 23068). Rampadarath et al. (2014) ont également montré que les extraits de J. multifida exercent une activité antibactérienne sur les souches de Bacillus algicola et $S$. epidermidis. L'approche toxicologique montre que l'extrait aqueux des feuilles de J. multifida provoque une stimulation à la consommation associée à une perte de poids. Normalement une augmentation de la consommation doit entrainer une accumulation de graisse et par conséquent un gain de poids. Mais nos expériences ont prouvé le contraire. L'augmentation de la consommation serait due à la stimulation de la production de l'hormone de la faim au niveau de l'intestin qui va envoyer un message au cerveau qui à son tour déclenche la sensation de faim (Cheylan, 2017). La perte de poids s'explique par le fait que $J$. multifida contiendrait des substances toxiques ayant des effets similaires aux effets provoqués par l'intoxication aux organophosphorés (Jacquet et al., 2019). En effet l'intoxication aux organophosphorés provoque les diarrhées, une paralysie musculaire, une sensation accrue. Ceci s'explique donc par la perte de poids observée chez les souris. En effet des études antérieures réalisées par Kollin \& Uziel (2006) ont montré que toutes les parties de J. multifida sont toxiques et précisément les graines. Ils ont isolé des substances toxiques telles: le curcin, la toxalbumine, l'huile purgative. Ils ont également prouvé que l'intoxication par J. multifida ressemble à l'intoxication aux organophosphorés (Kollin \& Uziel, 2006). Le fruit a été documenté contenir des toxines telles que la récine, la toxalbumine. L'ingestion de grandes quantités provoque des diarrhées sévères, la déshydratation, le choc et une insuffisance hépatique chez les enfants. La récine a également des effets cardiotoxiques, hémolytiques et plusieurs décès ont été rapportés d'elle (Kinghorn, 1991).

souris ont montré une toxicité subaigüe à $600 \mathrm{mg} / \mathrm{kg}$. Par conséquent, l'usage de J. multifida devrait être fait sur une courte durée et à doses modérées. Toutefois, des études toxicologiques approfondies doivent être menées sur cette plante afin de mieux élucider sa toxicité. 


\section{REMERCIEMENT}

Nous remercions les tradithérapeutes de la région maritime du Togo et tous ceux qui ont contribué de près ou de loin à la réalisation de cette étude.

\section{REFERENCES}

Adesola A and Adetunji A, 2007. L'efficacité de Jatropha multifida dans la gestion de la candidose orale : étude préliminaire. Le journal internet de la médecine alternative. 4 (1), 66-68.

Agban A, Karou DS, Tchacondo T, Atchou K, Batawila $\mathrm{K}, 2012$. Evaluation de l'activité antifongique des extraits de Cassia alata $L$. et de Piliostigma thonningii (Schum) Milne Redhead. Revue CAMES-Série A, 13(1).

Akharaiyi FC and Boboye B, 2010. Antibacterial and Phytochemical Evaluation of Three Medicinal Plants. Journal of Natural Products, $3: 27-34$.

Bruneton J, 2009. Pharmacognosie, phytochimie, plantes médicinales. $4 \mathrm{e}$ édition, $\mathrm{Ed}$. Tec et Doc, Paris, 1269 pp.

CASFM (Comité de l'Antibiogramme de la Société Française de Microbiologie), 2014. Recommandations, SFM, France, $59 \mathrm{pp}$.

Cheylan JM, 2017. Régulation de l'appétit et prise en charge des troubles alimentaires. Thèse de doctorat en Pharmacie, Faculté de Pharmacie de Marseille, Université d'Aix-Marseille II, France. $175 \mathrm{pp}$

Dougnon TV, Klotoé JR, Dougnon TJ, Sègbo J, Atègbo JM, Edorh PA, Sodipo O, Dougnon F, Dandjesso C, Loko F, Dramane K, 2012. Hemostatic activity screening and skin toxicity of sap of Jatropha multifida L. (Euphorbiaceae) used in traditional medicine (Benin). Asian Pacific Journal of Tropical Disease ; S927-S932.

Falodun A, Imieje V, Erharuyi O, Joy A, Langer P, Jacob M, Khan S, Abaldry M, Hamann M, 2014. Isolation of antileishmanial, antimalarial and antimicrobial metabolites from Jatropha multifida. Asian Pacific Journal of Tropical Biomedicine 4(5) : 374-378.

Fennell CW, Lindsey KL, McGaw LJ, Sprag SG, Staffort GI, Elgorashi EE, Grace OM, van Staden J, 2004. Assessing African medicinal plants for efficacy and safety: pharmacological screening and toxicity. Journal of Ethnopharmacology, 94 : 205-217.

Hamza OJ, van den Bout-van den Beukel CJ, Matee MI, Moshi MJ, Mikx FH, Selemani HO, Mbwambo ZH, Van der Ven AJ, Verweij PE,
2006. Antifungal activity of some Tanzanian plants used traditionally for treatment of fungal infections, Journal of Ethnopharmacology, 108 (1) : 124-132.

Harborne JB, 1998. Phytochemical methods : a guide to modern techniques of plant analysis. 3rd ed. London: Chapman \& Hall. ISBN : 0-41257270-2, $302 \mathrm{pp}$.

Hoekou YP, Tchacondo T, Karou SD, Koudouvo K, Atakpama W, Pissang $P$, Gbogbo KA, Woegan YA, Batawila K, Akpagana K, and Gbeassor M, 2016. Ethnobotanical Study of Latex Plants in the Maritime Region of Togo. Pharmacognosy Research, 8(2) : 128-134.

Jacquet $P$, Poirier L, Daudé D, Chabrière $E, 2019$. Intoxication aux organophosphorés : vers des traitements enzymatiques. Annales Pharmaceutiques Françaises, 77 : 349-362.

Jacques $F, 2002$. Flore illustré des phanérogames de Guadeloupe et de Martinique, Gondwana éditions, CIRAD ; Tome 2, 76-89.

Kaouadji M, Agban A, Mariotte AM, Tissut M, 1986. Lonchocarpene, a stilbene and Lonchocarpusone, an isoflavone: two new pyranopolyphenols from Lonchocarpus nicoun roots. Journal of Natural Products, 49(2) : 281285.

Kedziers A and Jouvelet B, 2006. "Guérisseurs et féticheurs: La médecine traditionnelle en Afrique de l'ouest. Editions Alternatives, 142 $\mathrm{pp}$.

Kinghorn AD, 1991. New techniques for the isolation and identification of phorbol esters and structurally related diterpene, in "Handbook of natural toxins, vol.6, Toxicology of plant and fungal coumpounds" Marcel Dekker, Inc., New York, pp.217-242.

Kollin D and Uziel Y, 2006. A case of Jatropha multifida poisoning resembling organophosphate intoxication. Journal of toxicology-clinical toxicology, 44: 337-338.

NCCLS, 2003. Methods for dilution antimicrobial susceptibility tests for bacteria that grow aerobically; approved standard. Sixth edition. Wayne, PA: NCCLS. NCCLS document no. M07-A6. 32 p. 
OMS, 2013. Stratégie de l'Organisation Mondiale pour la Santé pour la médecine traditionnelle pour 2014 - 2023. Available from http : //www.who.int/publications/list/traditional_medi cine_strategy/fr/, consulté le 25 juin 2017.

Pissang P, Tchacondo T, Hoekou YP, Maman I, Effoe S, Agban A, 2018. Toxicité subaigüe de Pteleopsis suberosa Engl. \& Diels (Combretaceae) : effet de l'heure d'administration chez la Souris. Afrique Science, 14(6) : $428-439$.

Rampadarath S, Puchooa D, Ranghoo-Sanmukhiya VM, 2014. Antimicrobial, phytochemical and larvicidal properties of Jatropha multifida Linn. Asian Pacific Journal of Tropical Medicine, 7(1) : S380-S383.

Soundararajan V, Zuraini Z, Yeng C, Lachimanan YL, Jagat RK, Sreenivasan S, 2012. The Antimicrobial Efficacy of Elaeis guineensis: Characterization, in Vitro and in Vivo Studies. Molecules, 17: 4860-4877.

Tittikpina NK, Agban A, Gbogbo KA, Hoekou YP, Pereki H, Batawila K, Akpagana K, 2013. Evaluation des propriétés antimicrobiennes de Pterocarpus erinaceus Poir (Faboideae) et Daniellia oliveri (Rolfe) Hutch. et Dalz (Caesalpinoideae), utilisées en médecine traditionnelle au Togo. International Journal of Biological and Chemical Sciences, 7(4) : 15861594. 